Revue internationale P.M.E.

Économie et gestion de la petite et moyenne entreprise

\title{
Diversité et unité des entreprises à forte croissance du secteur manufacturier en France
}

\section{Philippe Mustar}

Volume 14, numéro 3-4, 2001

URI : https://id.erudit.org/iderudit/1008698ar

DOI : https://doi.org/10.7202/1008698ar

Aller au sommaire du numéro

Éditeur(s)

Presses de l’Université du Québec

ISSN

0776-5436 (imprimé)

1918-9699 (numérique)

Découvrir la revue

Citer cet article

Mustar, P. (2001). Diversité et unité des entreprises à forte croissance du secteur manufacturier en France. Revue internationale P.M.E., 14(3-4), 67-89. https://doi.org/10.7202/1008698ar

\section{Résumé de l'article}

Cet article s'intéresse à la question des entreprises à forte croissance du secteur manufacturier en France. Faisant suite à un vaste travail d'analyse sur bases de données, les résultats présentés ici se fondent sur les réponses à un questionnaire postal adressé à près de 150 entreprises à forte croissance du secteur manufacturier en France. Dans une première partie, l'article montre que sous la dénomination commune de " PME à forte croissance " sont regroupées des PME aux trajectoires de croissance très diversifiées ; ces trajectoires sont elles-mêmes le résultat de stratégies différentes se déployant dans des contextes contrastés. Mais l'article souligne que, par-delà ces contextes stratégiques différents, plusieurs éléments communs jouent un rôle majeur dans le processus de la forte croissance. Ils concernent :

- les partenariats que forment ces entreprises avec des acteurs diversifiés pour innover ;

- la gestion de l'organisation particulière que constitue une entreprise à forte croissance;

- le financement de la croissance et le rôle des pouvoirs publics.

Ces éléments permettent à l'auteur de critiquer nombre de travaux sur les entreprises à forte croissance, travaux qui contiennent dans leur méthodologie de départ leurs résultats conclusifs. 


\title{
Diversité et unité des entreprises à forte croissance du secteur manufacturier en France ${ }^{1}$
}

\author{
Philippe MUSTAR \\ Centre de sociologie de l'innovation \\ École nationale supérieure des mines de Paris
}

\author{
MOTS CLÉS
}

PME - Forte croissance - Innovation - Pouvoirs publics Partenariats - Financement

\begin{abstract}
L'AUTEUR
PhILIPPE Mustar est professeur à l'École nationale supérieure des mines de Paris et chercheur au Centre de sociologie de l'innovation. Ses travaux participent à la construction d'une socioéconomie de la recherche et de l'innovation. Ils portent d'une part, sur les acteurs de l'innovation (et notamment les PME issues des institutions d'enseignement supérieur et de recherche) et, d'autre part, sur les politiques publiques de recherche et d'innovation. II vient de publier Research and Innovation Policies in the New Global Economy. An International Comparative Analysis, Edward Elgar, Cheltenham, UK, Brookfield, US, 509 p., 2002. Adresse : Centre de sociologie de l'innovation, École nationale supérieure des mines de Paris, 60, boul. Saint-Michel, 75006, Paris, France. Téléphone : 3314051 91 91, télécopieur : 331435456 28. Courriel : <mustar@csi.ensmp.fr>.
\end{abstract}

1. Cet article reprend quelques éléments d'une étude sur les entreprises à forte croissance du secteur manufacturier en France réalisée pour l'OCDE et financée par le ministère français de l'Industrie. Nous remercions tout particulièrement Mme Marie-Florence Estimé (OCDE) et M. Jean-Luc Vo Van Qui (DARPMI). Nous remercions l'ensemble des membres du groupe de travail de l'OCDE sur les PME et les membres du groupe d'experts qui ont fourni des remarques cruciales au cours de l'avancement de ce travail. Nous remercions notre collègue québécois, Pierre-André Julien, notamment pour ses commentaires très utiles sur notre questionnaire. Ce questionnaire a ensuite été retravaillé avec Philippe Crance de la société GMV qui est spécialisée dans les enquêtes auprès des entreprises et avec notre collègue Philippe Larédo (CSI). L'enquête postale et les relances ont été conduites par Martine Lecœur (GVM) ; nous les remercions tous ici. Enfin, nous remercions les deux évaluateurs anonymes qui ont permis d'améliorer ce texte. 


\title{
RÉSUMÉ
}

Cet article s'intéresse à la question des entreprises à forte croissance du secteur manufacturier en France. Faisant suite à un vaste travail d'analyse sur bases de données, les résultats présentés ici se fondent sur les réponses à un questionnaire postal adressé à près de 150 entreprises à forte croissance du secteur manufacturier en France. Dans une première partie, l'article montre que sous la dénomination commune de «PME à forte croissance » sont regroupées des PME aux trajectoires de croissance très diversifiées; ces trajectoires sont elles-mêmes le résultat de stratégies différentes se déployant dans des contextes contrastés. Mais l'article souligne que, par-delà ces contextes stratégiques différents, plusieurs éléments communs jouent un rôle majeur dans le processus de la forte croissance. Ils concernent:

- les partenariats que forment ces entreprises avec des acteurs diversifiés pour innover;

- la gestion de l'organisation particulière que constitue une entreprise à forte croissance;

- le financement de la croissance et le rôle des pouvoirs publics.

Ces éléments permettent à l'auteur de critiquer nombre de travaux sur les entreprises à forte croissance, travaux qui contiennent dans leur méthodologie de départ leurs résultats conclusifs.

\begin{abstract}
This article is on the issue of high-growth manufacturing firms in France. Following an extensive analysis of secondary data, the research results presented here were obtained from a postal survey of nearly 150 high-growth French SMEs. In the first part of the article, it is showed that under the guise of a common denomination, namely "high-growth SMEs", are very different growth trajectories. These trajectories are themselves the results of different strategies implemented in different contexts. However, beyond these different strategic contexts are common features that play a major role in the high-growth process. These are :

- the partnerships developed by these firms with various actors in order to innovate;

- the management of the specific organizational form constituted by the highgrowth firm;

- the financing of growth and the role of public bodies.
\end{abstract}

These features allows us to criticize previous work on high-growth enterprises in which results are predetermined by the methodology employed.

\section{RESUMEN}

Este trabajo trata sobre las empresas de fuerte crecimiento del sector industrial en Francia. La investigación es la continuación de un análisis en profundidad sobre la información contenida en diferentes bases de datos. Los resultados presentados proceden de las respuestas a una encuesta postal dirigida a cerca de 150 empresas de fuerte crecimiento del sector industrial en Francia. 
En la primera parte el artículo muesta que bajo la denominación común de «Pyme de fuerte crecimiento » se agrupan Pymes con trayectorias de crecimiento muy diversas. Éstas son el resultado de distintas estrategias desarrolladas en diferentes entornos. Más allá de los entornos estratégicos diferentes, el artículo pone de relieve que varios elementos comunes desempeñan un papel relevante en el proceso de fuerte crecimiento. Estos son:

- la cooperación de estas empresas con diferentes actores para innovar.

- la particular gestión de la organización que constituye une empresa de fuerte crecimiento.

- la financiación del crecimiento y el papel de las políticas públicas.

Estos elementos permiten a su autor realizar una crítica de numerosos trabajos sobre este tipo de empresas cuyas conclusiones están ya incluídas en la metodología de partida.

\section{ZUSAMMENFASSUNG}

Dieser Artikel beschäftigt sich mit stark wachsenden Produktionsunternehmen in Frankreich. Die breite Analyse basierte auf einer Datenbasis, wobei die aufgeführten Ergebnisse auf Antworten aus postalischen Fragebogen bei 150 stark wachsenden Produktionsunternehmen in Frankreich resultierten.

In einem ersten Teil zeigt der Artikel, dass hinter der Gruppierung mit der Beschreibung, KMU mit starkem Wachstum « Unternehmen mit unterschiedlichen Wachstumswegen erscheinen. Die Entscheidungswege sind selber das Resultat von verschiedenen Strategien, die sich in einem gegensätzlichen Kontext entfalten. Aber der Artikel unterstreicht, dass über die verschiedenen strategischen Kontexte hinaus, mehrere gemeinsame Elemente eine Hauptrolle spielen im Prozess des starken Wachstums. Sie betreffen :

- Die Partnerschaften, welche diese Unternehmen mit den verschiedenartigen Akteuren im Netz verbindet um innovativ tätig zu sein

- Das Organisationsmanagement, welches charakteristisch ist für stark wachsende Unternehmen

- Die Finanzierung des Wachstums und die Rolle der öffentlichen Macht.

Diese Elemente erlauben dem Autor viele Arbeiten über stark wachsende Unternehmen zu beurteilen. Es werden vor allem Arbeiten kritisiert, die schon in der Ausgangslage von klaren, beschliessenden Ergebnissen ausgehen.

\section{Introduction}

Nombre de travaux récents ont souligné, à des degrés divers et parfois avec de fortes controverses, le rôle des PME dans la création d'emplois (Birch, 1981; Carree et Klomp, 1996; Caballero, Engel et Haltiwanger, 1997 ; Davidsson, 1995, 1989 ; Davidsson, Lindmark et Olofsson, 1998 ; Davis, Haltiwanger et Schuh, 1996; Kirchhoff et Phillips, 1988 ; Julien, 2000 ; OCDE, 1994 à 1998b). L’une de ces

Revue internationale P.M.E., vol. 14, nos 3-4, 2001 
controverses se manifeste autour de la question suivante : quelles PME contribuent majoritairement à la création de nouveaux emplois? Pour certains, tels Storey (1994), Birch (1981) ou Birch, Haggerty et Parsons (1993), un petit groupe de PME à très forte croissance joue un rôle capital et serait la source de la majorité des nouveaux emplois. À l'inverse, d'autres avancent que la création d'emplois est principalement le résultat de la création et du développement incrémental de très nombreuses petites nouvelles entreprises (Davidsson, Lindmark et Olofsson, 1995). Voilà posés les termes du débat qui oppose les tenants des « gazelles » aux partisans des « souris » (gazelles versus mice). Ces dernières années, l'attention des hommes politiques comme celles des universitaires s'est principalement portée sur ces jeunes entreprises en création et notamment sur celles qui connaissent une forte croissance (Baldwin, Chandler et Papailiadis, 1994 ; Baldwin et Johnson, 1996). Sans mésestimer leur importance, nous proposons de porter le regard sur une autre réalité : sur les PME déjà existantes ou plus anciennes (que nous appelons PME permanentes ${ }^{2}$ par opposition aux PME en création ou nouvellement créées), et notamment sur celles qui connaissent, elles aussi, une forte croissance de leurs effectifs salariés.

Ces entreprises ont fait l'objet de peu d'attention. Ainsi dans son étude, Birch analyse trois groupes d'entreprises auxquels il donne des surnoms d'animaux : les éléphants (les grandes entreprises cotées en Bourse), les souris (les petites entreprises qui démarrent et croissent très lentement) et les gazelles (les petites firmes qui démarrent et qui ont une forte croissance). Aucune place spécifique n'est faite aux entreprises moyennes et anciennes.

Cet article reprend quelques-unes des conclusions du travail que nous avons mené au sein du groupe de travail de l'OCDE sur les PME à forte croissance. À la base de notre travail, nous avons fait le triple constat suivant : $a$ ) à côté des nouvelles entreprises à forte croissance existe un ensemble de firmes permanentes à forte croissance elles aussi ; $b$ ) que ce groupe d'entreprises permanentes à forte croissance a fait l'objet de peu d'intérêt (ces entreprises restent dans une zone d'ombre. Elles sont mal connues, par exemple en ce qui concerne le type de croissance qu'elles connaissent) ; c) enfin, que l'étude fine des entreprises appartenant à ce groupe peut relever d'un double intérêt : théorique, pour bâtir une meilleure théorie des firmes à forte croissance, et pratique, pour prendre des mesures politiques permettant de soutenir ces entreprises et d'en augmenter le nombre. Du point de vue des politiques

2. Explicitation du terme «entreprise permanente »: la recherche porte sur le secteur de l'industrie manufacturière en France. En 1994, ce secteur comptait près de 23000 entreprises de plus de 20 salariés. Notre travail s'intéresse aux entreprises qui, au sein de cette population, existaient déjà en 1985. Autrement dit, nous analysons les entreprises permanentes durant la période allant de 1985 à 1994. Sont donc exclues les entreprises qui ont été créées pendant ces dix années, celles qui ont disparu ou celles dont le nombre de salariés est passé sous la barre des 20 salariés.

Revue internationale P.M.E., vol. 14, nos 3-4, 2001 
publiques, l'idée que nous défendons est qu'il n'y a pas une opposition mais une complémentarité entre les nouvelles entreprises à forte croissance et les entreprises plus anciennes qui connaissent, elles aussi, une forte augmentation de leurs effectifs.

Notre travail sur les entreprises à forte croissance du secteur manufacturier en France comportait deux phases. La première a consisté en différentes analyses quantitatives à partir des bases de données du ministère chargé de l'Industrie (bases de données qui contiennent des informations sur près de 23000 entreprises de plus de 20 salariés). Il s'agissait de décrire l'importance de ces entreprises et de tracer les différents types de trajectoires que pouvait prendre la croissance. La deuxième phase de l'étude avait pour objectif de mettre au jour les principales caractéristiques de ces entreprises à forte croissance et les facteurs liés à la dynamique de croissance qu'elles ont connue. Cette seconde phase a reposé sur une enquête postale détaillée qui a permis d'analyser 143 entreprises à forte croissance.

Cet article propose une lecture transversale des résultats de la deuxième phase (les résultats complets de l'étude ont été publiés de façon détaillée dans un rapport de l'OCDE (OCDE, à paraître) $)^{3}$.

L'hypothèse générale qui a guidé notre travail est que la forte croissance est un processus, mais que ce processus n'est pas unique, qu'il n'y a pas une meilleure façon de faire, mais plutôt différentes configurations contrastées de forte croissance. Les 500 entreprises à forte croissance que nous avons repérées et étudiées dans la première phase sont diverses; et il paraît illusoire de vouloir trouver « la » recette qui conduirait au succès ou une formule qui permettrait de reproduire le succès ou les exemples de réussite exceptionnelle. Mais, au-delà de leur forte hétérogénéité et des différentes configurations dans lesquelles elles se déploient, les entreprises à forte croissance ont certaines caractéristiques communes. L'objectif de cet article basé sur cette enquête postale est de mettre au jour certains éléments que l'on retrouve par-delà les configurations variées de la croissance.

\section{Méthodologie}

\subsection{La problématique}

Les résultats de nos analyses quantitatives (Mustar, 2001), l'analyse des études menées dans différents pays sur les PME à forte croissance, la lecture des travaux récents en économie industrielle, en sociologie et en gestion de l'innovation, les demandes du ministère français chargé de l'Industrie et de l'OCDE, les entretiens

3. Nous suggérons au lecteur intéressé par la comparaison de la population d'entreprises décrite dans cet article avec celle plus large de l'enquête sur bases de données - dite de la première phase - de consulter ce rapport, notamment pour évaluer les effets de nonréponse à notre enquête postale.

Revue internationale P.M.E., vol. 14, ns 3-4, 2001 
que nous avons réalisés avec de nombreux dirigeants de PME ayant connu une forte croissance..., tous ces éléments ont permis de dégager les quatre thèmes qui sont apparus comme centraux pour notre analyse.

1. La caractérisation processus de croissance. Pour comprendre ce processus, il est nécessaire de disposer d'informations sur : la trajectoire de la croissance (est-elle linéaire? se fait-elle par étapes ? se passe-t-elle sur une durée courte ou sur un grand nombre d'années ?...) La forme de la croissance: est-elle le fruit de fusions et d'acquisitions (c'est-à-dire d'une croissance externe) ou d'un développement interne de l'entreprise ? Son caractère sectoriel : la croissance se passe-t-elle dans le même secteur ou l'entreprise estelle plutôt obligée de sortir de son secteur d'origine (Birley et Westhead, 1990) ? Ses relations avec le marché : la croissance repose-t-elle sur les mêmes produits ou services ou sur de nouveaux produits, de nouveaux services ? La géographie de la croissance : celle-ci est-elle nationale, européenne, mondiale ?

2. La dynamique des réseaux et des partenariats. L'hypothèse qui préside à cet ensemble de questions est que le processus de croissance est lié à des alliances et à des partenariats avec différents types d'acteurs : quelle est la dynamique de ces réseaux? Quelles alliances apparaissent dans le domaine de la production? Les clients, les consommateurs ou les usagers sont-ils intégrés à ce processus de croissance? Quels partenariats observet-on dans le domaine de la technologie et de l'innovation? Quels liens existent avec les universités ou les organismes publics de recherche ? Quels partenariats commerciaux observe-t-on?

3. L'organisation de la firme, son management (Barringer, Jones et Lewis, 1998), ses ressources humaines. L'hypothèse qui nous guide ici est qu'une PME à forte croissance doit recruter et se réorganiser constamment. Comment le fait-elle ? Quelles sont les formes d'organisation et leurs évolutions ? Quelles sont les compétences et les ressources humaines nécessaires? Quelles sont les principales formes de management? Comment la stratégie évolue-t-elle?

4. Le rôle de l'intervention publique. Nos enquêtes préalables sur le cas français montrent une présence importante des pouvoirs publics dans le processus de croissance. Qui intervient (ministères, agences publiques, régions, Commission européenne, etc.) ? Dans quels domaines interviennent les pouvoirs publics (exportations, technologie, aide au recrutement, etc.) ? De quelle façon (subventions, avances remboursables, prêts, conseils, etc.) ? Pour répondre à ces questions, il est nécessaire de disposer d'un ensemble d'éléments que les bases de données que nous avons utilisées jusqu'à présent ne contiennent pas. Aussi, avons-nous fait le choix de mener une enquête par questionnaire postal.

Revue internationale P.M.E., vol. 14, $\mathrm{n}^{\text {os }} 3-4,2001$ 
Ces quatre thèmes sont fortement interdépendants et sont loin d'épuiser la totalité des questions que soulèvent les entreprises à forte croissance. Par exemple, nous ne traitons pas de la problématique des qualités de l'entrepreneur individuel. Il existe une abondante documentation sur l'entrepreneur et ses qualités propres ; cet aspect important n'est pas abordé dans notre étude. Nous avons en effet choisi de concentrer notre analyse sur la dynamique de l'entreprise en forte croissance plutôt que sur les qualités de l'entrepreneur, cela tant pour des raisons théoriques que pour des raisons pratiques. Nos différents travaux antérieurs ont souligné que la création ou le développement d'une entreprise à forte croissance était le résultat d'un processus collectif. La dynamique de l'entreprise est le fruit d'un travail d'équipe et des complémentarités qui lui sont liées. D'un point de vue théorique, connaître les caractéristiques d'un seul membre de cette équipe, en fût-il le dirigeant principal, n'offre qu'un intérêt limité ; cette façon de faire est même contreproductive puisque qu'elle revient à nier les aspects collectifs du processus que nous souhaitons étudier. D'un point de vue pratique, connaître les caractéristiques de l'équipe tout entière exigerait un travail spécifique et une enquête lourde qui dépasseraient le cadre de cette étude centrée sur l'entreprise. L'étude menée par P.-A. Julien au Québec apporte des éléments de réponse aux différentes questions concernant l'entrepreneur et ses qualités.

\subsection{Le questionnaire postal}

À partir des quatre blocs de questions, un questionnaire a été élaboré. Sa construction a été particulièrement difficile pour deux raisons. Premièrement, c'est un questionnaire très large. En effet, les thèmes abordés sont multiples. Réaliser un questionnaire sur un thème précis (par exemple les PMI et l'innovation ou un autre sur les PMI et l'exportation) aurait été plus simple. Deuxièmement, c'est un questionnaire qui vise non pas à réaliser une photographie à un moment donné, mais à saisir certains éléments d'une dynamique : le processus de croissance de l'entreprise sur une dizaine d'années.

Une première version du questionnaire a été envoyée aux différents experts nationaux participant à l'étude. De nombreuses réunions de travail et les discussions que nous avons eues nous ont permis de simplifier un grand nombre de questions, de les rédiger dans un langage moins savant et plus proche de celui des managers d'entreprises. Des questions d'opinions ont été ajoutées afin de donner un rythme plus détendu au questionnaire. Une attention particulière a été apportée à la forme et à la mise en page du questionnaire. Au total, nos quatre thèmes principaux ont été subdivisés en 10 rubriques : les caractéristiques de la croissance, le processus de croissance, les partenariats, l'organisation, les ressources humaines, la technologie, le marché, le marketing, le financement de la croissance et le rôle des pouvoirs publics.

Revue internationale P.M.E., vol. 14, nos 3-4, 2001

(C) 2002 - Presses de l'Université du Québec

Édifice Le Delta I, 2875, boul. Laurier, bureau 450, Sainte-Foy, Québec G1V 2M2 • Tél. : (418) 657-4399 - www.puq.uquebec.ca

Tiré de : Revue internationale P.M.E., vol. 14, nos 3-4, sous la direction de Pierre-André Julien. 
Le questionnaire a ensuite été testé auprès d'un échantillon d'une dizaine d'entreprises. Il s'agissait de rencontrer le dirigeant d'une société à forte croissance et de lui faire remplir le questionnaire en restant à ses côtés et en observant ses réactions, ses hésitations, ses difficultés. Cette phase du test a été essentielle, car elle nous a révélé les limites de notre questionnaire qui demandait des informations sur une période d'une dizaine d'années : la majorité des responsables d'entreprises nous ont dit ne plus se souvenir précisément de l'état des choses il y a dix ans et nous ont avertis qu'ils ne consulteraient pas leurs archives pour nous répondre. Cela nous a obligés à revoir l'ensemble des questions portant sur la dynamique, à les simplifier en les rendant plus qualitatives. De nouveaux tests ont été réalisés pour mesurer la pertinence de ces simplifications.

\subsection{La population interrogée}

Ayant déjà pratiqué ce type d'enquête postale, nous savions que les taux de retour étaient faibles. Si nous voulions pouvoir traiter un nombre de réponses significatives, il fallait adresser le questionnaire à un nombre important d'entreprises. Près de 1000 entreprises ont été sélectionnées dans les bases de données que nous avions précédemment utilisées dans le cadre de notre travail avec l'OCDE (2001). Ces entreprises sont celles qui ont connu la plus forte croissance de leur effectif (mesurée à la fois en termes absolus et relatifs grâce à un indicateur qui combine la différence d'employés entre les années de début et de fin de période en volume et le ratio du nombre d'employés en fin de période sur le nombre en début de période ${ }^{4}$ parmi les 23000 entreprises de plus de 20 salariés que compte le secteur manufacturier en France). Au total, ces 1000 entreprises ont une activité industrielle, ont connu une forte croissance entre 1988 et 1998 (forte par rapport aux autres entreprises de ce secteur) et existaient donc déjà dans la seconde moitié des années 1980 (sans que l'on ne mette un seuil pour leur date de création).

\subsection{Analyse des répondants à l'enquête postale}

Le questionnaire a été envoyé au principal dirigeant de chacune de ces entreprises. Les réponses spontanées ont été peu nombreuses : moins d'une trentaine. Une importante campagne de relance téléphonique a été engagée, des centaines d'entreprises ont été recontactées et un nouvel exemplaire du questionnaire leur a été adressé. En définitive, ce sont 143 questionnaires exploitables qui nous ont été retournés après plusieurs mois de travail. Les personnes qui ont effectué les relances - et qui ont donc souvent dialogué avec des chefs d'entreprise - expliquent ce taux de réponse relativement faible (proche de $15 \%$ ) par deux raisons principales :

4. Cf. Rapport OCDE, op. cit. 
- les entreprises reçoivent certainement trop de questionnaires et nombreuses sont celles qui nous ont répondu que si cette enquête n'était pas obligatoire, elles n'y répondraient pas ;

- de nombreuses entreprises qui avaient connu une forte croissance pendant la période 1985-1994 (parfois même dans la première partie de cette période) ne se reconnaissaient plus comme des firmes à forte croissance en 1999 au moment de l'enquête postale; elles nous ont dit ne pas être concernées.

C'est généralement le principal dirigeant de l'entreprise qui nous a retourné le questionnaire ; mais ce questionnaire a souvent été rempli par plusieurs personnes dans l'entreprise.

Un tiers des entreprises qui ont répondu à notre questionnaire appartiennent, en début de période, à la classe des moins de 50 salariés. Le taux de croissance du nombre moyen de salariés est, pour les 143 entreprises qui ont répondu à notre questionnaire, de 2,4. L'effectif moyen des entreprises analysées par l'enquête postale est de 158 salariés en début de période (en général 1988); il passe à 373 salariés en 1998. Le nombre moyen de salariés a été multiplié par 2,4. Mais le tiers de ces entreprises a multiplié son nombre de salariés par plus de trois; et un sixième de ces entreprises l'a multiplié par plus de cinq.

TABLEAU 1

Répartition des répondants par classe de taille au début de la période

\begin{tabular}{lc}
\hline $\begin{array}{l}\text { Taille } \\
\text { (salariés) }\end{array}$ & $\begin{array}{c}\text { Questionnaires } \\
\mathbf{1 4 3} \text { entreprises (en 1988*) }\end{array}$ \\
\hline $20-49$ & $33 \%$ \\
$50-99$ & $17 \%$ \\
$100-249$ & $29 \%$ \\
$250-499$ & $14 \%$ \\
+500 & $7 \%$ \\
\hline
\end{tabular}

* Début de la période sur laquelle portait le questionnaire, la fin de la période étant 1998.

Les quatre sections qui suivent reprennent les principaux résultats des quatre thèmes sur lesquels était basé notre questionnaire. 


\section{Trajectoires et marches de la croissance}

Les entreprises qui ont répondu à notre questionnaire appartiennent au secteur manufacturier. Elles sont réparties entre différents sous-secteurs ${ }^{5}$ et localisées dans tout l'Hexagone $^{6}$. En fin de période, $55 \%$ des répondants sont devenus des filiales de groupe.

\subsection{La représentation graphique de la croissance}

Notre questionnaire propose quatre trajectoires de croissance et demande aux entreprises de choisir dans laquelle elles se reconnaissent. Il offre également une case vide pour les entreprises qui préfèrent dessiner elles-mêmes la courbe qu'a suivie la croissance de leurs effectifs salariés. Les entreprises qui ont fait ce choix ont été soit reclassées dans un des quatre groupes (parce que la courbe dessinée est semblable ou proche de l'une des courbes proposées), soit regroupées dans un cinquième groupe. Ce cinquième groupe est relativement homogène : il comprend une vingtaine d'entreprises qui enregistrent une première phase de croissance, puis une forte chute et une nouvelle phase de la croissance.

Le type 1, qui représente une croissance plutôt continue sur la période, regroupe $22 \%$ des entreprises. En début de période, près de la moitié des entreprises appartenant à ce groupe ont moins de 50 salariés. En moyenne, ces entreprises ont 145 salariés au début de la période et 500 salariés à la fin. Ce sont celles qui ont connu la plus forte croissance; leurs effectifs ont été en moyenne multipliés par 3,4.

Le type 2 est celui où la croissance se fait sur un nombre limité d'années. Le nombre de salariés est stable, puis une croissance a lieu durant quelques années. Ensuite, le nombre de salariés redevient stable. Ce groupe compte $15 \%$ des entreprises. Plus de la moitié d'entre elles ont plus de 100 salariés en début de période. En moyenne, ces entreprises ont 180 salariés au début de la période et 375 salariés à la fin ; leurs effectifs ont été en moyenne multipliés par 2,1.

Le type 3, celui de la croissance chaotique, regroupe $33 \%$ des firmes. En moyenne, ces entreprises ont 160 salariés au début de la période et 290 salariés à la fin. Leurs effectifs ont été en moyenne multipliés par 1,8.

5. Compte tenu de la taille réduite de la population qui a répondu à notre questionnaire, nous ne répartirons pas dans notre analyse les 143 entreprises entre 15 sous-secteurs.

6. Compte tenu de la petite taille de l'échantillon, nous n'utiliserons pas non plus une répartition entre les 22 régions. 


\section{Les cinq trajectoires types de croissance}

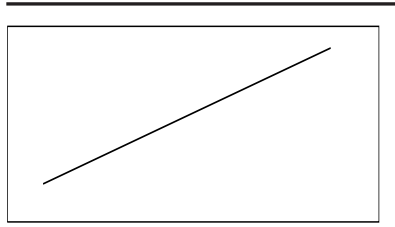

Type $1(22 \%)$

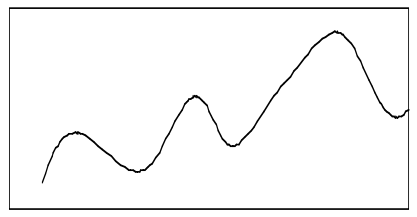

Type $3(33 \%)$

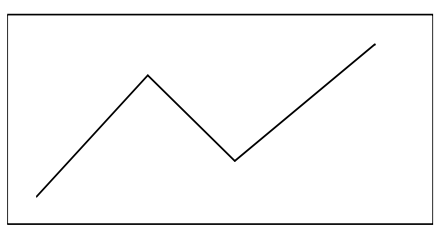

Type $5(13 \%)$

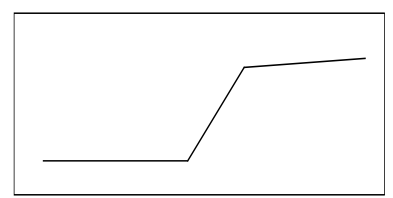

Type $2(15 \%)$

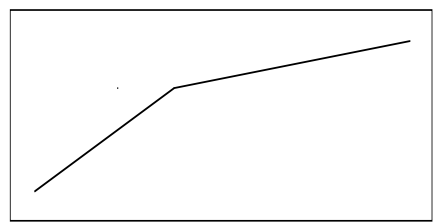

Type $4(17 \%)$

Le type 4, marqué par une croissance forte dans la première partie de la période, puis par une croissance ralentie dans la seconde partie, regroupe $17 \%$ des entreprises. En début de période, $70 \%$ de ces entreprises ont moins de 100 salariés. En moyenne, ces entreprises ont 100 salariés au début de la période et 290 salariés à la fin. Leurs effectifs ont été en moyenne multipliés par 2,8.

Enfin, le type 5 regroupe $13 \%$ des firmes qui connaissent une période de croissance, puis une chute, puis une reprise. En moyenne, ces entreprises ont 225 salariés au début de la période et 450 salariés à la fin. Leurs effectifs ont été en moyenne multipliés par 2.

La rareté relative de la croissance continue, même pour les entreprises très performantes que nous avons relevées (référence OCDE), est confirmée. Ici, on note également un fléchissement de la croissance dans les groupes 3, 4 et 5. Nous pouvons faire l'hypothèse qu'il marque les effets de la crise de 1993. Les entreprises en forte croissance sont, comme les autres, sensibles à la conjoncture. 


\subsection{L'exportation : un préalable à la forte croissance}

L'enquête postale révèle que plus des trois quarts des entreprises en forte croissance exportent. Pour un peu plus du quart de ces entreprises qui exportent, l'exportation représente moins de $10 \%$ du chiffre d'affaires. Pour un peu plus d'un autre quart, l'exportation fournit entre $10 \%$ et $33 \%$ du chiffre d'affaires. Pour un peu moins de la moitié des entreprises, l'exportation compte pour plus du tiers du chiffre d'affaires.

Ce résultat s'accorde avec les conclusions de nombreuses études qui établissent une relation entre exportation et forte croissance. Nos deux enquêtes (celleci par questionnaire et celle que nous avons menée sur les bases de données) soulignent également le fait que la part des firmes qui exportaient déjà au départ de la période est importante : on ne peut pas dire que c'est l'exportation qui tire la croissance.

L'exportation n'est pas le point d'arrivée du processus de la forte croissance, mais apparaît plutôt comme un point de départ du processus de croissance, une sorte de préalable. L'exportation - et l'ouverture sur d'autres marchés, d'autres circuits de distribution et d'autres clientèles - anticipe plutôt qu'elle ne suit la forte croissance, elle l'accompagne plus qu'elle ne la tire. Même si, suivant leur taille de départ, les entreprises ont des comportements différents à l'égard de l'exportation : plus l'entreprise est petite, plus la part de son chiffre d'affaires réalisé à l'exportation augmente. C'est dans les entreprises les plus importantes que cette part est la plus grande, tant en début qu'en fin de période. Mais c'est aussi dans ces entreprises que l'évolution de cette part est la plus faible.

Pour connaître la croissance, un marché national porteur est tout aussi important que l'exportation. Il est en effet nécessaire de relativiser l'importance de l'exportation : elle procure en moyenne un tiers du chiffre d'affaires. Des marchés nationaux porteurs sont, dans la majorité des cas, indispensables à la forte croissance.

Enfin, nous avons également noté que, pour ces entreprises, il est nécessaire d'être très près de leurs clients, d'être en relation directe avec eux. Pour cela, près de la moitié d'entre elles ont créé des filiales commerciales à l'étranger.

\subsection{Forte croissance ne rime pas avec diversification sectorielle}

Nos enquêtes ont montré que la croissance se fait très majoritairement dans le même secteur d'activité ; elle est rarement le résultat d'une diversification. Les exemples d'entreprises qui se sont engagées dans des secteurs d'activité parfois très éloignés de leurs marchés traditionnels et qui ont alors connu une forte croissance existent. Mais cette figure de la forte croissance reste minoritaire : seule une entreprise sur 
quatre déclare avoir réalisé une diversification en termes de secteur d'activité. Dans trois cas sur quatre, la croissance se fait dans le même secteur d'activité ; mais à l'intérieur de ce même secteur, l'entreprise a été innovatrice.

\subsection{Les marchés des entreprises à forte croissance ne sont pas seulement des marchés en forte croissance}

Un tiers seulement des marchés de ces entreprises sont des marchés en forte croissance. Plus de la moitié des entreprises en forte croissance œuvrent sur des marchés en faible croissance. On retrouve là le fait qu'une minorité d'entreprises a répondu - au début du questionnaire - de façon négative à la question : «La croissance est-elle fondée sur une croissance naturelle du marché ? » La croissance n'est pas tirée «naturellement» par le marché, mais réclame une stratégie proactive. Enfin, dans un cas sur six, les entreprises ont connu une forte croissance sur des marchés sans croissance ou en récession.

Dans la quasi-totalité des entreprises en forte croissance, la croissance s'est faite par la conquête de nouveaux clients. L'élargissement de la gamme de produits ou de prestations vendus aux mêmes clients a aussi joué pour la presque totalité des entreprises. Les deux principales façons de faire semblent nécessaires simultanément pour connaître la croissance : élargir la gamme de produits que l'on vend aux mêmes clients et toucher de nouveaux clients sont deux stratégies plus complémentaires que concurrentes.

\subsection{Une majorité d'entreprises à forte croissance sont liées à quelques clients principaux par un ou deux produits phares}

Comment les entreprises en forte croissance commercialisent-elles leurs produits ? Près de 9 entreprises en forte croissance sur 10 commercialisent leurs produits ou services elles-mêmes directement; cette façon de faire leur donne un contact immédiat avec les clients. On retrouve là le rôle important des clients qui agissent souvent comme des partenaires. La concentration du chiffre d'affaires avec les principaux clients est grande: les deux tiers des entreprises à forte croissance dépendent de leurs cinq principaux clients, qui leur procurent entre le quart et la totalité de leur chiffre d'affaires.

La part de marché moyenne en France du produit phare des entreprises en forte croissance est de plus de $50 \%$, celle de leur second produit, de $33 \%$. En moyenne, ces deux produits phares des entreprises en forte croissance assurent plus de la moitié de leur chiffre d'affaires réalisé en France. Une population d'entreprises à forte croissance vit sur ses deux premiers produits. Une grande partie de ces entreprises ne peuvent aller plus en avant sur ces mêmes marchés et doivent chercher ailleurs une diversification ou une extension géographique.

Revue internationale P.M.E., vol. 14, $\mathrm{n}^{\text {os }} 3-4,2001$ 


\section{Dynamique des partenariats et de l'innovation}

\subsection{La croissance est fortement liée à la mise en place de partenariats en tout genre}

L'enquête postale a mis en exergue le rôle central des partenariats dans le processus de forte croissance. La quasi-totalité des chefs d'entreprise interrogés soulignent que la forte croissance réclame des partenariats. Dans le réseau de l'entreprise à forte croissance, un partenaire majeur apparaît : le client (cités dans 8 cas sur 10). C'est principalement avec des clients que les entreprises en forte croissance ont formé des partenariats.

Un deuxième partenaire majeur est composé - pour les entreprises filiales par «les autres entités du groupe » auquel l'entreprise appartient. Deux tiers des filiales déclarent avoir réalisé leur croissance en partenariat avec d'autres entités du groupe auquel elles appartiennent.

Derrière ces deux partenaires majeurs, on peut dessiner deux autres cercles de partenariats. Le premier cercle concerne les distributeurs, les fournisseurs et les sous-traitants (qui sont cités entre une fois sur quatre et une fois sur trois). Le second cercle de partenaires comprend les prestataires de service et les concurrents (cités par près de 1 répondant sur 6), les centres techniques industriels et les entreprises de recherche (cités 1 fois sur 10) et les laboratoires publics (cités par 1 entreprise sur 20).

Moins nombreuses sont les entreprises qui semblent avoir créé des partenariats dans le domaine de la recherche. Dans le questionnaire, nous demandions aux entreprises si elles avaient participé à des programmes de recherche impliquant des collaborations entre différents pays, si elles avaient participé à des programmes de recherche avec des laboratoires publics ou si elles avaient des projets européens en cours. On peut ajouter à ces entreprises celles qui ont répondu par l'affirmative aux questions sur les partenariats avec des entreprises de recherche, avec des centres techniques industriels et avec des laboratoires publics. Au total, si l'on tient compte de ces multiples formes possibles de coopération avec la recherche, une entreprise à forte croissance sur trois a établi des partenariats dans le domaine de la recherche.

Pourquoi les entreprises tissent-elles des partenariats? Ce n'est pas tant pour partager les risques et les coûts que les entreprises coopèrent que pour ouvrir de nouveaux marchés et mettre au point de nouveaux produits, services ou procédés. Si les entreprises à forte croissance établissent des partenariats, c'est bien pour lever les incertitudes techniques dans la mise au point de nouveaux produits ou procédés et pour ouvrir de nouveaux marchés. 


\subsection{L'innovation de produit est un moteur de la forte croissance}

Les entreprises qui ont connu une forte croissance ont adopté une stratégie volontariste d'innovation en surmontant les incertitudes techniques (elles ont mis au point de nouveaux produits) et les incertitudes commerciales (elles ont réussi à conquérir de nouveaux marchés et à élargir géographiquement leur marché). L'étude a confirmé le rôle clé de l'innovation de produit (près de 8 entreprises sur 10 relient la croissance à la mise au point des produits et services nouveaux ou à l'élargissement de la gamme de produits et de services). Très majoritairement, les entreprises en croissance sont restées dans leur secteur d'origine (dans trois cas sur quatre), mais elles ont élargi leur zone géographique d'action et leur gamme de produits; elles ont innové en développant des produits et des services nouveaux.

\subsection{L'innovation dans les entreprises à forte croissance s'appuie sur des projets de R-D (qui ne correspondent pas toujours aux définitions classiques de la recherche)}

Les entreprises à forte croissance sont beaucoup plus fortement engagées que les autres dans des activités de recherche soit de manière directe ou indirecte. L'enquête postale montre que près de 6 entreprises en forte croissance sur 10 déclarent posséder une activité de recherche organisée. En moyenne, près de $5 \%$ du chiffre d'affaires de ces entreprises est consacré à la recherche et plus de $7 \%$ de leurs salariés sont employés aux activités de R-D. L'effort de recherche est plus important qu'il y a dix ans pour plus de 7 entreprises en forte croissance sur 10.

Des entreprises qui déclarent ne pas avoir de recherche organisée déposent des brevets ou bénéficient du crédit d'impôt en faveur de la recherche (cela parce que la procédure du crédit d'impôt recherche permet de prendre en considération les dépenses de recherche externe ou sous-traitée). Si l'on tient compte de ces entreprises, ce sont, en définitive, 7 entreprises à forte croissance sur 10 qui sont engagées dans des activités de recherche.

Les entreprises en forte croissance font de la R-D de façon différente de celle qui est reconnue par les définitions strictes de l'activité de R-D et nombreuses sont celles qui n'ont pas de R-D organisée au sens de Frascati. Pour les filiales, le groupe auquel l'entreprise appartient apporte compétences et résultats de recherche. Enfin, notons que l'activité de R-D de ces firmes a souvent bénéficié du soutien des pouvoirs publics.

Il en va ainsi pour les entreprises qui ont de la R-D organisée. Ainsi, 70\% d'entre elles ont bénéficié du crédit d'impôt recherche durant leur période de croissance.

Revue internationale P.M.E., vol. 14, $\mathrm{n}^{\text {os }} 3-4,2001$ 


\section{Gérer l'organisation innovante en forte croissance}

\subsection{Gérer l'organisation innovante}

Les responsables des entreprises à forte croissance ne gèrent pas seulement l'innovation, ils gèrent une organisation innovante. L'innovation dans les entreprises à forte croissance est un acte total. Elle ne concerne pas que les produits nouveaux, elle s'accompagne d'une innovation de processus et de changements dans l'organisation de l'entreprise et dans la commercialisation de ses produits.

Le système de production des entreprises à forte croissance est familier des techniques de gestion de production sophistiquées. En effet, plus de 7 entreprises en forte croissance sur 10 ont mis en place une gestion de production assistée par ordinateur (GPAO). Les deux tiers de ces entreprises ont une certification ISO et un système de flux tendu. Pour près de 7 entreprises sur 10, les modifications du système de production ont contribué à la croissance. Si la forte croissance se caractérise par l'innovation de produit, cette dernière n'est pas séparable de l'innovation de processus qui, elle aussi, contribue à la croissance.

Dans ces entreprises à forte croissance du secteur manufacturier, on notera le poids important de la production et la montée des activités de conception. Un long processus de changement se met en place, dont la progression réelle semble avoir échappé à de nombreux analystes. Cette rapide expansion des activités de méthodes et de bureau d'études joue un rôle important dans les entreprises que nous avons suivies.

Pour les dirigeants des entreprises interrogés, la forte croissance est plus liée à l'innovation de processus et à l'innovation de produit qu'à l'innovation commerciale. Cette dernière n'est cependant pas négligeable puisqu'elle est présente dans le tiers des entreprises que nous étudions.

La grande majorité des entreprises en forte croissance ont, au cours de leur croissance, connu des changements d'organisation. Nous avons jusqu'à présent noté que les entreprises en forte croissance ont modifié leurs produits et leur processus de production et qu'elles ont modifié leur système de commercialisation. Il n'est dès lors pas étonnant que 7 entreprises en forte croissance sur 10 déclarent avoir connu des changements d'organisation. À cet égard, les entreprises indépendantes ne se distinguent pas de façon significative des filiales.

Les entretiens que nous avons eus avec des responsables de société en croissance au cours de cette étude renforcent l'idée que le phénomène de croissance est intimement lié à la capacité d'innovation de l'entreprise. Mais ils permettent également de poser l'hypothèse que la durabilité de la forte croissance est liée à la capacité de la firme à enclencher un processus d'innovation tourbillonnaire. Le processus d'innovation tourbillonnaire est celui qui donne naissance, de façon

Revue internationale P.M.E., vol. 14, $\mathrm{n}^{\text {os }} 3-4,2001$ 
continue ou régulière, à des innovations de produit, à des innovations de production ou à des innovations organisationnelles (généralement ces trois types d'innovation se développent simultanément). Le modèle tourbillonnaire s'oppose à une conception de l'innovation conçue comme un processus permettant de passer d'une situation stable à une autre situation stable. Il s'oppose à la logique du « coup », du «one shoot». Ce système permet de réassembler des compétences techniques ou commerciales que maîtrise l'entreprise avec des éléments nouveaux. Avec le modèle tourbillonnaire, il n'y a plus un point de départ et un point d'arrivée, mais des améliorations en continu du produit (améliorations incrémentales, sortie de nouvelles versions, développement de gammes), du système de production, de la commercialisation et, plus généralement, de l'organisation même de la firme.

\subsection{Les entreprises à forte croissance ont une organisation largement ouverte sur l'extérieur qui n'hésite pas à faire appel à des compétences externes}

Plus des deux tiers des entreprises en forte croissance procèdent à une veille concurrentielle systématique. Trois entreprises à forte croissance sur quatre procèdent également à une mesure de la satisfaction de leurs clients. On retrouve là l'attention importante apportée aux clients, déjà soulignée. Pour mieux appréhender leurs marchés, les entreprises en forte croissance ont recours à différentes façons de faire. Cependant, les agents commerciaux de l'entreprise jouent le rôle particulièrement crucial de médiateurs entre les activités de conception de l'entreprise et les utilisateurs de ses produits. En effet, pour connaître leurs marchés, 8 entreprises en forte croissance sur 10 recourent aux rapports de vendeurs. L'enquête a également montré que ces entreprises à forte croissance utilisent de nombreuses méthodes et maîtrisent de nombreux outils de marketing.

Plus d'une entreprise sur deux a eu recours à des consultants. C'est principalement aux services de spécialistes en organisation qu'elles font appel (plus qu'à des consultants en marketing ou en technologie et innovation).

L'ouverture sur l'extérieur des entreprises à forte croissance se retrouve dans les modalités de recrutement des principaux cadres de la firme. Les principaux cadres des entreprises en forte croissance ont, dans les deux tiers des entreprises, été recrutés à l'extérieur. Dans un tiers des cas, ils sont issus de la promotion interne.

Enfin, les deux tiers des entreprises en forte croissance procèdent également à une activité de veille technologique systématique. Et à l'époque où elles ont répondu à notre questionnaire, une large majorité d'entre elles maîtrisait les technologies électroniques nouvelles de communication: elles disposaient d'un site Internet et une très large majorité utilisait le courrier électronique pour communiquer avec leurs clients et leurs partenaires extérieurs, mais aussi pour la communication interne à l'entreprise.

Revue internationale P.M.E., vol. 14, $\mathrm{n}^{\text {os }}$ 3-4, 2001 


\subsection{Les entreprises à forte croissance accordent une grande importance à la formation de leur personnel et font bénéficier ce personnel des bénéfices de la croissance}

Le pourcentage de la masse salariale consacré par les entreprises en forte croissance à la formation a augmenté pour la quasi-totalité d'entre elles sur la période. Pour la moitié d'entre elles, il a même fortement augmenté. Au total, près de $4 \%$ de la masse salariale de ces entreprises est consacré à la formation, ce qui est supérieur à la dépense moyenne des PME françaises.

L'intéressement est une pratique courante dans les entreprises à forte croissance. Dans près de 8 entreprises en forte croissance sur 10, les salariés ont un intéressement. Parmi ces entreprises, 9 fois sur 10, cet intéressement concerne la totalité des salariés. Rappelons que, en moyenne, l'intéressement n'est pratiqué que dans $10 \%$ des entreprises de l'industrie en France. La pratique de l'intéressement serait donc un signe distinctif des entreprises en forte croissance dans des secteurs très différents et prendrait des formes très variées.

\section{Financement de la croissance et rôle des pouvoirs}

La croissance est un processus et ce processus a un coût. Les entreprises le financentelles facilement? Avant d'aborder les modalités de financement de ce coût, notons que plus des deux tiers des dirigeants d'entreprises en forte croissance qui ont répondu à notre questionnaire sont « tout à fait d'accord » ou «d'accord» avec les opinions suivantes :

- «ce n'est pas lors de la création que le financement pose le plus de problèmes, c'est après »;

- «le système bancaire français n'est pas adapté au financement des entreprises en forte croissance »;

- «c'est très difficile de financer la croissance en conservant son indépendance ».

Cette dernière constatation est particulièrement prégnante puisque, nous l'avons signalé, une entreprise à forte croissance sur deux est filiale d'un groupe en fin de période.

\subsection{Comment les entreprises financent-elles leur croissance?}

L'enquête postale a permis de hiérarchiser différentes modalités de financement. Notons d'abord que les formes de financement liées aux marchés financiers à risque sont peu courantes pour ces entreprises permanentes du secteur manufacturier: l'arrivée de nouveaux actionnaires est citée dans moins de 1 cas sur 10, le recours au capital-risque et le marché boursier sont cités chacun dans 1 cas sur 20.

Revue internationale P.M.E., vol. 14, $\mathrm{n}^{\text {os }} 3-4,2001$ 
La source de financement la plus courante est interne à la firme : c'est le résultat de l'entreprise qui finance ou qui participe au financement de la croissance. Le résultat de l'entreprise est une source de financement de la croissance citée par quasiment toutes les entreprises. Mais elle n'exclut pas d'autres sources.

Trois autres sources de financement, externes cette fois, sont fortement citées : ce sont l'emprunt, les soutiens des pouvoirs publics et le groupe auquel éventuellement l'entreprise appartient.

L'emprunt est cité par une entreprise sur deux. Ensuite, et de façon plus inattendue, les subventions et les soutiens publics sont cités par une entreprise sur trois. De façon assez surprenante, l'intervention des pouvoirs publics joue un rôle important dans le processus du financement de la croissance dont nous allons détailler les modalités ci-après. Enfin, un tiers des entreprises appartenant à un groupe a bénéficié d'apports financiers de ce groupe pour sa croissance.

Pour une majorité d'entreprises permanentes du secteur manufacturier, l'adossement à un grand groupe est un point de passage obligé du processus de croissance.

Pour les entreprises filiales d'un groupe, le questionnaire demandait : «Le groupe auquel vous appartenez a-t-il contribué à la croissance ? » Dans près de 6 filiales sur 10, le groupe a contribué à la croissance. Dans un cas sur deux, par un apport en compte courant et en augmentation de capital. Puis, dans moins d'un cas sur deux, en diffusant des bonnes pratiques et en mettant à disposition des savoir-faire techniques; enfin, dans un peu moins d'un cas sur quatre, en mettant à disposition du personnel et des moyens de recherche.

\subsection{Le rôle des pouvoirs publics}

Les pouvoirs publics jouent un rôle crucial dans le financement du processus de croissance. Les entreprises savent à la fois identifier et utiliser la panoplie des soutiens publics.

L'enquête a montré que les pouvoirs publics jouent un rôle crucial dans le processus de financement des PME à forte croissance (à côté du financement par les résultats de l'entreprise ou par l'emprunt). Les entreprises en forte croissance ont jugé très utiles les aides diverses au processus d'innovation tels que :

- les avances remboursables et les aides à l'embauche de l'ANVAR, les soutiens des DRIRE (ministère chargé de l'Industrie);

- les subventions des collectivités locales (conseils régionaux, conseils généraux, municipalités, etc.);

Revue internationale P.M.E., vol. 14, $\mathrm{n}^{\text {os }} 3-4,2001$ 
- le soutien de la Coface pour l'exportation ou les programmes de la Commission européenne pour la technologie ;

- enfin, comme nous l'avons déjà signalé, le crédit d'impôt en faveur de la recherche.

Pour la plupart des entreprises, ces aides sont cruciales, mais il faut les considérer comme un accompagnement et non pas comme un déclencheur de la croissance. Notons un point positif: très largement, les chefs d'entreprise ne pensent pas que les aides publiques soient compliquées à obtenir, mais plusieurs reprochent à ces soutiens de manquer de continuité.

On peut conclure que, dans le cas français, les entreprises que nous avons interrogées savent à la fois identifier et utiliser la panoplie des soutiens publics disponibles.

\section{Conclusion, enseignements pratiques, théoriques et méthodologiques}

Dans une première partie, nous avons montré que la dénomination commune de «PME à forte croissance » vise des trajectoires de croissance très diversifiées, qui sont elles-mêmes le résultat de stratégies différentes se déployant dans des contextes contrastés. Nous avons également souligné que par-delà ces contextes stratégiques différents, plusieurs éléments jouent un rôle majeur dans le processus de la forte croissance. Ils concernent :

- les partenariats que tissent ces entreprises avec des acteurs diversifiés pour innover;

- la gestion de l'organisation particulière que constitue une entreprise à forte croissance;

- le financement de la croissance et le rôle des pouvoirs publics.

De nombreuses questions restent posées. Deux points demandent notamment des analyses plus fines. Le premier concerne les différences de comportement et de facteurs de succès des PME filiales et des PME indépendantes (par exemple sur la question des exportations et des éventuelles transactions internes aux groupes; Boccara, 1997). Le second est lié aux relations avec des acteurs diversifiés que nous avons regroupés sous le terme générique de partenariat. Que recouvrent ces partenariats? Quelles formes prennent-ils ? Qu'est-ce qui circule entre les acteurs engagés (financements, compétences, objets techniques, textes, etc.) ? (Callon, 1991.) Mais, à côté de ces travaux complémentaires à mener et sur la base des résultats présentés ci-dessus, nous souhaitons conclure cet article par la question méthodologique de l'analyse de la forte croissance. 
En effet, les études sur les entreprises à forte croissance ne s'intéressent qu'aux cas exceptionnels : des firmes où la croissance est strictement continue sur la période étudiée, des firmes qui, en début ou en fin de période, ne sont pas des filiales de grands groupes, des firmes qui ne connaissent qu'une croissance organique (toute fusion ou acquisition est éliminée), des firmes où les dirigeants détiennent, au moins en partie, le capital, etc.

De cette manière, ces études (qui par exemple ne prennent en considération que les firmes qui connaissent $20 \%$ de croissance annuelle pendant cinq ans) ne s'intéressent qu'à un type très particulier d'entreprises. Elles donnent ainsi une image assez lisse de la réalité où les conflits n'existent pas : tous les ans, les ventes ou le nombre de salariés grimpe et la trajectoire de l'entreprise à forte croissance s'apparente à un long fleuve tranquille. Les histoires que ces études nous racontent sont «sans chair», elles laissent de côté la quasi-totalité des entreprises pour lesquelles la forte croissance est faite de chocs successifs (changement de dirigeant, réorganisations fortes, etc.).

Cela est gênant d'un point de vue théorique : comment bâtir une théorie de l'entreprise en croissance en ne s'intéressant qu'à des cas marginaux ? Mais aussi d'un point de vue pratique: comment les politiques publiques peuvent-elles être efficaces si elles se basent sur une réalité fortement biaisée, si elles ne tiennent compte que des cas où les agents sont beaux, riches, intelligents et en bonne santé ? Cela ne veut pas dire que l'on ne puisse pas tirer des enseignements de ces cas de réussite exceptionnelle; mais ces enseignements ne seront valables que dans certaines configurations bien particulières qui ne sont pas celles où se trouvent l'immense majorité des entreprises.

Ces études que nous critiquons posent aussi problème aux responsables des entreprises : si l'on accepte l'image de la croissance continue qu'elles donnent, gérer la croissance revient alors à gérer la continuité. Or, comme celle-ci est marginale, les enseignements risquent fort d'avoir peu de pertinence - voire d'être contreproductifs - pour l'entreprise prise dans le monde réel.

En définitive, nos analyses des trajectoires des entreprises à forte croissance montrent que la forte croissance est un processus, mais que ce processus n'est pas unique, qu'il n'y a pas de «bonne recette », mais plutôt différentes configurations contrastées de forte croissance. Cela renforce la double idée suivante : que l'on ne peut pas prendre comme critère de croissance le taux de croissance pour une année donnée comme le font de nombreuses études et qu'il est nécessaire d'étudier ce phénomène dans la durée pour comprendre les formes que prennent les trajectoires de la croissance. Du point de vue des politiques publiques, il semble qu'elle doive éviter d'avoir une définition trop élitiste de la PME en croissance; elles doivent retenir que la croissance prend des formes diversifiées.

Revue internationale P.M.E., vol. 14, $\mathrm{n}^{\text {os }} 3-4,2001$ 


\section{Bibliographie}

BALDWIN, J.R., C. CHANDLER et T. PAPAILIADIS (1994), Stratégies pour le succès. Le profil des PME en croissance au Canada, Ottawa, Statistique Canada, $\mathrm{n}^{\circ}$ 61-523ER.

BALDWIn, J.R. et J. Johnson (1996), Innovator Typologies and Strategies of Success, Ottawa, Statistique Canada, juillet.

BARRINGER, B.R., F.F. JONES et P.S. LEWIS (1998), « A qualitative study of the management practices of rapid-growth firms and how rapid-growth firms mistigate the managerial capacity problem », Journal of Developmental Entrepreneurship, vol. 3, $\mathrm{n}^{\circ} 2$, p. $97-140$.

BIRCH, D. (1981), «Who creates jobs? », The Public Interest 65.

BIRCH D., A. Haggerty et W. PARsons (1993), Who's Creating Jobs?, Waltham, Cognetics Inc.

BIRLEY, S. et P. WESTHEAD (1990), «Growth and performance constrasts between "types" of small firms », Strategic Management Journal, vol. 11, n 7, p. 535-557.

BocCARA, F. (1997), «Mythes et réalités sur l'emploi et la croissance des PME : le rôle des groupes (1984-1992)», Document de travail, INSEE.

CABAllero, Ricardo, E.M.R.A. Engel et John Haltiwanger (1997), « Aggregate employment dynamics : building from microeconomic evidence », American Economic Review, mars.

CAllon, M. (1991), «Réseaux technico-économiques et irréversibilités», dans R. Boyer, B. Chavance et O. Godard (dir.), Les figures de l'irréversibilité en économie, Paris, Édition de l'École des hautes études en sciences sociales, p. 195-230.

CARree, M. et L. Klomp (1996), «Small business and job creation : a comment », Small Business Economics, vol. 8.

DAVIDSSON, P. (1989), «Entrepreneurship and after? A study of growth willingness in small firms », Journal of Business Venturing, vol. 4, no 3, p. 211-226.

DAVIDSSON, P. (1995), «SMEs and job creation in Sweden», Communication présentée au séminaire OCDE de haut niveau sur «Les PME: l'emploi, l'innovation et la croissance », Washington, D.C.

DAVIDSSON, P., L. LINDMARK et C. OLOFSSON (1995), « Smallness, newness and regional development », Communication présentée au séminaire OCDE de haut niveau sur «Les PME : l'innovation et l'emploi », Washington, D.C.

DAVIDSSON, P., L. LINDMARK et C. OLOFSSON (1998), «The extent of overestimation of small firm job creation - an empirical examination of the regression bias », Small Business Economics, vol. 11.

DAVIS, S., J.C. HALtiwanger et S. SchuH (1996), Job Creation and Destruction, Cambridge, MIT Press.

JuliEN, P.-A. (2000), L'entrepreneuriat au Québec de 1980 à 2005, Montréal, Éditions Transcontinental; Québec, Fondation de l'entrepreneurship. 
KIM, W.C. et R. MAUBorgne (1997), «Value innovation: the strategic logic of highgrowth », Harvard Business Review, vol. 75, n 1, p. 102-111.

KIRCHHOFF, B.A. et B.D. PHILLIPS (1988), «The effect of firm formation and growth on job creation in the United States », Journal of Business Venturing, vol. 3, $\mathrm{n}^{\mathrm{0}} 3$, p. 261-272.

Ministry OF ECONOMIC AFFAIRS OF THE NETHERLANDS (1999), High-Growth Companies in the Netherlands.

MustaR, P. (2002), «High-growth manufacturing firms in France», dans High-Growth SMEs : Their Contribution to Employment and Their Characteristics, OCDE, Paris (à paraître).

OCDE (1994), The OECD Jobs Study, Paris.

OCDE (1996), Technology, Productivity and Job Creation, Paris.

OCDE (1996), OECD Employment Outlook, Paris.

OCDE (1997a), Implementing the OECD Jobs Strategy : Member Countries' Experience, Paris.

OCDE (1997b), Small Business, Job Creation and Growth: Facts, Obstacles and Best Practices, Paris.

OCDE (1998a), Fostering Entrepreneurship, Paris.

OCDE (1998b), Technology, Productivity and Job Creation : Best Policy Practices, Paris.

OCDE (2002), High-Growth SMEs : Their Contribution to Employment and Their Characteristics, Paris, à paraître.

PEnRose, E.T. (1959), Facteurs, conditions et mécanismes de la croissance des entreprises, traduction de son ouvrage par S.L. Mayret, Paris, Les Éditions Hommes et Techniques, 1963.

SESSI (1998), L'innovation technologique dans l'industrie, Paris.

SESSI (1998), Les compétences pour innover dans l'industrie, Paris.

STOREY, D. (1994), «Understanding the small business sector », Londres, Routledge.

STOREY, D. (1995), The Ten Percenters, Rapport préparé pour la firme Andersen \& Cie, Londres.

Storey, D. (1997), The Ten Percenters, Londres, Deloitte \& Touche.

Revue internationale P.M.E., vol. 14, nºs 3-4, 2001 